\title{
Methodology and Models in Erosion Research: Discussion and Conclusions
}

\author{
R.P. Shellis ${ }^{a, b} \quad$ C. Ganss ${ }^{c} \quad$ Y. Ren ${ }^{d}$ D.T.Zero ${ }^{\text {e }}$ A. Lussi ${ }^{b}$ \\ ${ }^{a}$ School of Oral and Dental Science, University of Bristol, Bristol, UK; ${ }^{b}$ Department of Preventive, Restorative and

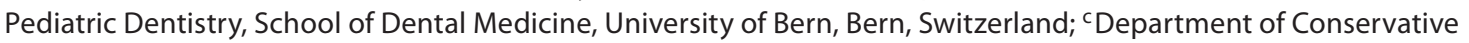 \\ and Preventive Dentistry, Dental Clinic, Justus Liebig University Giessen, Giessen, Germany; ${ }^{\text {dUniversity of }}$ \\ Rochester Eastman Institute for Oral Health, Rochester, N.Y., and e Department of Preventive and Community \\ Dentistry, Indiana University School of Dentistry, Indianapolis, Ind., USA
}

\section{Key Words}

Dental erosion • Enamel softening • Erosion models •

Erosive wear $\cdot$ Hardness measurements $\cdot$ Intra-oral

erosion $\cdot \mathrm{pH}$

\begin{abstract}
This paper summarises the discussions which took place at the Workshop on Methodology in Erosion Research in Zürich, 2010, and aims, where possible, to offer guidance for the development and application of both in vitro and in situ models for erosion research. The prospects for clinical trials are also discussed. All models in erosion research require a number of choices regarding experimental conditions, study design and measurement techniques, and these general aspects are discussed first. Among in vitro models, simple (single- or multiple-exposure) models can be used for screening products regarding their erosive potential, while more elaborate $\mathrm{pH}$ cycling models can be used to simulate erosion in vivo. However, in vitro models provide limited information on intra-oral erosion. In situ models allow the effect of an erosive challenge to be evaluated under intra-oral conditions and are currently the method of choice for short-term testing of low-erosive products or preventive therapeutic products. In the future, clinical trials will allow longer-term testing. Possible methodologies for such trials are discussed.
\end{abstract}

Copyright $\odot 2011$ S. Karger AG, Basel
(C) 2011 S. Karger AG, Basel

$0008-6568 / 11 / 0457-0069 \$ 38.00 / 0$

Fax +4161306 1234

E-Mail karger@karger.ch

www.karger.com
Accessible online at:

www.karger.com/cre
This paper summarises the discussions which took place at the Workshop on Methodology in Erosion Research in Zürich, 2010, and aims, where possible, to offer guidance for future studies.

Following the presentations on special topics which form the basis of the preceding papers, the participants were divided into two groups. The first group, chaired by A. Lussi and D.T. Zero, consisted of M. Buzalaf, J.A. Cury, R.P. Shellis, L.M.A. Tenuta and A. Young (reporter), and discussed initial erosion models. The second group, chaired by C. Ganss and Y. Ren, consisted of T. Attin, D.W. Bartlett (reporter), M.S. Duggal, R.P. Ellwood, A.S. Hara, M.C.D.N.J.M. Huysmans, N. Schlueter, N.X. West and $\mathrm{A}$. Wiegand, and discussed substance loss models. In a plenary session, the chairs of the discussion groups summarised the respective findings and the participants had the opportunity to add their comments. Finally, the discussion moved on to consider the prospects for clinical studies.

In erosive wear, the tooth surface is first attacked by acid, and the resulting loss of structural integrity and mechanical strength, termed 'softening' by Koulourides [1968], renders the surface vulnerable to intra-oral abrasive forces, including forces that would have no significant effect on a sound tooth surface. In their contribution to this Workshop, Huysmans et al. [2011] proposed that the term 'erosion' should be used for the softening effects of acid on the tooth surface, while the subsequent wear process induced by softening should be termed 'erosive tooth 
wear'. There are obvious advantages in a terminology which distinguishes between the two aspects of this wear process, but the definitions proposed by Huysmans et al. [2011] may require modification, because enamel loss can result from a prolonged erosive challenge without involvement of abrasion. Available evidence suggests that erosive challenges in vivo are brief, and it is thus perhaps unlikely that erosion alone is a major direct cause of surface loss in real life. However, this wear process can certainly be achieved in experimental models. Therefore, we suggest that the term 'erosion' should include not only the initial partial demineralisation (which is adequately covered by Koulourides's term 'softening') but also any surface loss caused solely by extended exposure to acid. This terminology does have the disadvantage that until now 'erosion' has been commonly used as shorthand for 'dental erosion' to describe the whole process from softening to tissue loss. In geology, the chemical dissolution processes contributing to erosion are termed 'corrosion', and this might be a more suitable term to describe the effects of acids in dental erosion. In the present paper, however, we use the terms 'erosion' and 'erosive tooth wear' to refer to the chemical and chemical-mechanical processes, as defined above.

The second discussion group at the Workshop considered that surface loss (erosive tooth wear) models should include only those in which both erosive and abrasive challenges were applied. The outcome variable in dental erosion research is either the damage caused by an acid challenge or the tissue loss caused by a combination of acid challenge and abrasion (in both cases, with or without remineralisation). The acid challenges in models of dental erosion are frequently sufficient to cause tissue loss without the involvement of abrasion. Therefore, in this paper, for the purpose of discussion, models are divided into erosion-only and erosion-abrasion (or erosive-wear) models. Many points made in separate discussions at the Workshop related to more than one of the models. As there was substantial agreement on these points, the discussion here commences with a consideration of these general aspects. Special aspects of erosion-only and erosion-abrasion models are then discussed, and the paper concludes with a summary of the discussion on clinical studies.

\section{General Aspects of Erosion Models}

\section{Specimen Preparation and Treatment}

Substrate

The substrate should be hard tissue derived from human or bovine teeth. It was considered that human tis- sues are the substrate of choice, but the increasing difficulty in procuring an adequate supply of human teeth was recognised. Evaluation of the available evidence led to the conclusion that, while human enamel and dentine are the substrates of choice, bovine tissues are suitable for most purposes. They are easier to acquire and should provide valid relative data, even if they cannot be extrapolated directly to the human situation.

Artificial substrates, such as hydroxyapatite discs, are considered suitable only for exploratory in vitro studies but not for in situ studies.

\section{Specimen Preparation}

However specimens of hard tissue are prepared, it is important in both in vitro and in situ models to carefully define the baseline specimen condition. As far as possible, the polishing procedure should be standardised, as should the depth of material removed in preparing a flattened surface. If necessary, samples should be stored in moist conditions between studies or between cycles. Overnight or long-term storage in a humid chamber in the presence of thymol crystals is recommended. Tests have shown that specimens show no change in micro- or nanohardness when thus stored for several days [Lussi, unpubl. data].

\section{Acid Treatment}

In general, the acid challenge should be appropriate for one of the main clinical problems: exposure to either an erosive soft drink or gastric reflux. In all in vitro models and in most in situ models where the acid challenge is administered ex vivo, choices about several variables that affect the extent of erosion have to be made. These key variables are:

(a) Erosive Agent. For modelling of gastric erosion, the only practical option is to use a solution of hydrochloric acid of appropriate concentration and $\mathrm{pH}$ [Young and Tenuta, 2011]. For modelling of extrinsic agents, there is a choice between a soft drink or fruit juice on the one hand and a simple acid solution on the other hand. Soft drinks offer the advantage of more realistic testing but may vary between batches. This could be a disadvantage in a series of studies performed over a long time. Soft drinks can also contain substances (e.g. sugars, gums, and polyphenols) which could modify the properties of the pellicle. Simple acid solutions are reproducible, inexpensive and can be formulated to produce a consistent response. Citric acid is usually used to simulate a soft drink acid, but other acids such as malic, acetic or lactic could also be appropriate, depending on the hypothesis of the study. The concentration of the acid which, together with the 
$\mathrm{pH}$, determines the buffer capacity should be similar to that in the erosive challenge being modelled.

(b) Positive Control. To check methodology (often referred to as a 'positive control'), inclusion of a standard acid solution is desirable. For this purpose, a solution of $0.052 \mathrm{~mol} / \mathrm{l}(1.0 \%)$ citric acid, $\mathrm{pH} \mathrm{3.6}$, seems suitable, which has a $\mathrm{pH}$ within the range of 3.5-3.75 for orange juice and the same titratable acidity. For a more severe challenge, the same solution, but at its natural $\mathrm{pH}$ (2.14 at $25^{\circ} \mathrm{C}$ ), could be used.

(c) $p H$. The $\mathrm{pH}$ of the erosive agent should be within the relevant range for acidic drinks, other agents or gastric reflux fluid, depending on the aim of the study [Young and Tenuta, 2011].

(d) Agitation. The flow rate of solution across the specimen surface should be controlled in a reproducible and preferably quantitative manner. To achieve this, a calibrated stirrer [Hemingway et al., 2008] or a chamber through which the erosive solution is pumped at a known rate [Attin et al., 2003] can be used.

(e) Constancy of Composition. A volume-to-substrate ratio large enough to ensure that conditions do not change during the course of the erosive challenge should be used. Preliminary experiments to establish an appropriate ratio will be required. For instance, the concentrations of calcium and phosphate could be monitored to check whether the degree of saturation does not increase measurably.

(f) Temperature. Since it can significantly affect erosion rate, temperature should be controlled, e.g. by use of a water bath or incubator. Body temperature $\left(37^{\circ} \mathrm{C}\right)$, average intra-oral temperature $\left(36^{\circ} \mathrm{C}\right)$ or 'room temperature' could be used. As the latter varies geographically, seasonally and over the course of a day, it is suggested that a controlled temperature of $25^{\circ} \mathrm{C}$ should be adopted for 'room temperature'.

(g) Duration of Erosive Challenge. The duration of an erosive challenge will depend on the variables in a-c and e above but, like other variables, should fall within a clinically appropriate range depending on the challenge being investigated. There was no absolute consensus on the duration of the challenge, but it was recognised that in vivo most acid challenges, from either gastric or dietary sources, did not last for more than a few minutes. The duration of the challenge will probably have to be adjusted to suit the type of substrate being used, as bovine enamel and dentine might differ from human dental hard tissues with respect to acid susceptibility.

It was considered essential that the experimental details with respect to these parameters be reported in full detail when the results are published.

\section{Method of Assessment}

Methods of assessing erosion have been reviewed several times [Barbour and Rees, 2004; Attin, 2006; Schlueter et al., 2011]. To assess enamel softening, the most useful method is to measure microhardness. Both Knoop and Vickers indenters have been used. However, because of their greater depth/length ratio, Vickers indenters will tend to penetrate softened enamel more deeply, possibly to the extent that indentation depth could be unduly influenced by the underlying sound enamel, which would result in a loss of sensitivity. Thus, Vickers indenters should be used with small loads, e.g. $10 \mathrm{~g}$, but to increase accuracy, it might be necessary to use special equipment (such as the Fisherscope) rather than standard hardness testers to measure the resulting small indentation lengths.

To obtain accurate results, specimens should have flat, polished surfaces, but less accurate measurements on natural surfaces might have potential for in vivo use [Schlueter et al., 2011]. In the earliest stages of softening, nanoindentation provides data on hardness and elastic modulus. Use of nanoindentation in conjunction with atomic force microscopy allows mechanical changes to be correlated with topography and ultrastructure. Analysis of calcium and phosphate release to the erosive solution can also provide information on the earliest stages of erosion. If this method is used in conjunction with small sample volumes (to improve analytical sensitivity), both calcium and phosphate should be analysed and the $\mathrm{Ca} / \mathrm{P}$ ratio in the solution should be calculated in order to make sure that no re-precipitation has occurred.

Where surface loss has occurred, single post-erosion hardness measurements are inappropriate, as the softening either reaches a steady state value (in erosion-only models) or the softened layer is removed (in erosion-abrasion models). For assessing the earliest stages of tissue loss due to abrasion, an indentation can be made after the erosive challenge, and reduction in its length following abrasion gives a direct measure of erosion depth, as there is a fixed relationship between indenter length and depth. The accuracy of this method is much reduced if erosion is performed after making the indentation, as acid attack will deepen the indentation [Attin, 2006]. Knoop indenters are to be preferred because they have a long and a short axis, whereas Vickers indenters are symmetrical. Profilometry, preceded by removal of demineralised matrix in the case of dentine, is suitable for assessing all stages of tissue loss within the limits of instrument precision. Microradiography is an alternative method but requires expensive equipment, and transversal microradiography is also destructive. 
The accuracy of any profilometer under the experimental conditions must be reported. Manufacturers provide accuracy of profilometers under ideal conditions, but these rarely reflect the conditions used in any dental investigation. Therefore, although the ultimate accuracy of a machine is useful information, it does not provide sufficient information on the conditions used in an investigation.

For in vivo studies, surface loss has been studied on replicas of test teeth using a variety of surface mapping techniques [Schlueter et al., 2011] including white-light interferometry [Holme et al., 2005]. Further development of replica-based methods for quantifying erosion in vivo would be valuable.

A variety of microscopic and surface analysis techniques is available for closer examination of structural and chemical changes at the tooth surface [Schlueter et al., 2011]. For in vivo studies, qualitative information about the surface microstructure can be obtained by scanning electron microscopy of high-quality replicas.

\section{In vitro Models}

Specimen Pre-Treatment

In vivo, tooth surfaces are usually covered with an acquired salivary pellicle, except after recent exposure to an erosive challenge, which tends to remove the greater thickness of the pellicle, leaving only the thin basal layer [e.g. Hannig et el., 2009]. In view of the erosion-inhibiting effect of the pellicle, it is considered desirable to expose specimens to saliva for a period before exposure to an acid challenge in order to simulate in vivo conditions. One in vitro study suggested that exposure of enamel to saliva for $1 \mathrm{~h}$ or more is necessary for the development of a pellicle which is protective against erosion, but for dentine a few minutes' exposure would suffice [Wetton et al., 2006].

An important question is whether it is better to use saliva from one donor or to use pooled saliva taken from several individuals. The former approach can be justified as ensuring consistency and the latter as avoiding bias: the protective effect of in vitro pellicle seems to vary considerably between individuals [Wetton et al., 2007]. However, the composition of saliva changes with the degree and method of stimulation, with the stimulation time and with the time of day. It is therefore crucial to standardise the method and time of collection no matter whether one or several donors are involved.

To overcome problems of the natural variability of saliva, Fujikawa et al. [2008] used pooled natural saliva which had been dialysed against a standard inorganic artificial saliva. Their particular protocol can be criticised on the grounds of excessive dialysis time and use of an inadequate antibacterial. Moreover, the authors provided no evidence from electrophoresis as to the composition of the dialysed saliva, and it is not known what type of pellicle it would form [Z. Cheaib, pers. commun.]. Nevertheless, the concept is interesting and could be the basis for producing a medium close to natural saliva with a standardised electrolyte composition.

On the basis of published work [Carlén et al., 1998], it can be questioned whether saliva applied in vitro produces a pellicle which truly resembles pellicle in vivo. This question merits further research.

An important aspect of the use of human saliva is the problem of controlling cross-infection. The use of disposable containers is recommended where possible. In experiments involving elaborate apparatus such as wear machines, which are difficult to disinfect, artificial saliva should be used, even though it is less than ideal.

\section{Screening and Basic Erosion Models}

These may employ either single or multiple acid challenges. In multiple-exposure models, specimens are stored in a humid atmosphere between challenges rather than in a remineralising medium as in a cycling model.

\section{Cycling Models}

The aim of models of this type is to assess the effect of storage in a remineralising solution to simulate possible repair processes in vivo. Either one cycle or several may be used. The cycling can be achieved either by manual transfer between media or by more elaborate, automated systems, usually termed artificial mouth models.

The duration of cycling experiments does not need to be as long as in caries research because erosion is a more rapidly progressing condition and even early lesions, created by erosive challenges that simulate clinical conditions, can be quantified reliably by available methods. The pattern of cycling of any acidic challenge should reflect a dietary habit or medical disorder and remain clinically relevant. Among the variables to be considered are: the duration of each challenge, the number and timing of challenges, and the timing of any treatment. The summary tables prepared by Young and Tenuta [2011] and Wiegand and Attin [2011] provide useful preliminary information for planning experiments, and specific recommendations are provided by Wiegand and Attin [2011] for the erosive challenge in substance loss studies.

For the remineralising medium, artificial saliva is in most cases acceptable. However, it should be remembered that in vivo some salivary proteins bind calcium, so that artificial saliva formulations based on the electrolyte 
composition of natural saliva but lacking salivary proteins are likely to have too high a degree of supersaturation and could be prone to precipitate excessive amounts of calcium phosphate. It is recommended that the degree of saturation of artificial saliva used in erosion experiments should be adjusted to match the results of Larsen and Pearce [2003], who corrected calcium concentrations for calcium binding. A further complication is that in natural saliva, some proteins (e.g. statherin) tend to inhibit calcium phosphate precipitation. It is difficult to model this in artificial formulations, but it should be borne in mind that remineralisation in vitro is likely to be greater than in vivo.

For experiments in which pellicle formation is part of the cycle, storage in natural human saliva is not necessarily essential: for relatively short periods (overnight or during weekends), use of a humid chamber is acceptable.

\section{In situ Models}

All clinical studies require ethical approval and should follow Good Clinical Practice guidelines. Oral and written instructions to study participants should be supplied and efforts made to check the compliance of the volunteers to the protocol. Reporting of in situ studies should follow the CONSORT statement [Schulz et al., 2010] where appropriate.

\section{Substrate}

The tooth specimens must be sterilised and/or conform to local ethical standards. A wide variety of methods for sterilisation or disinfection of specimens has been used. Ethylene oxide gas and $\gamma$-radiation do not alter surface microhardness or the response to demineralisation of enamel [Chandler, 1990; Amaechi et al., 1998, 1999]. Sterilisation by these methods must be performed by specialist companies and is relatively expensive. Methods that can be used in ordinary laboratories include autoclaving, dry heat sterilisation and immersion in formalin, povidoneiodine, $70-75 \%$ ethanol or sodium hypochlorite solutions. Not all of these methods have been fully tested with respect to both bactericidal efficiency and their effect on the hard tissues. Amaechi et al. [1998] reported that autoclaving, povidone-iodine and sodium hypochlorite sterilised specimens but affected surface appearance, although they caused no significant increases in artificial caries lesion formation. Lippert et al. [2004] found that sodium hypochlorite (13\%, 3 days) treatment did not affect enamel nanohardness. Viana et al. [2010] recently reported encouraging results with microwave irradiation, which appeared to affect neither surface microhardness nor the re- sponse to demineralisation or remineralisation. In summary, available information suggests that some methods are effective sterilising agents with no effect on the properties of enamel. However, more information in both respects is needed on a number of methods in current use.

Not all methods appropriate for enamel may be suitable for use with dentine. $\gamma$-Irradiation sterilises dentine effectively without altering its properties [White et al., 1994; Preston et al., 2007; Brauer et al., 2008]. Other methods, e.g. autoclaving and $0.1 \%$ aqueous thymol solution, were effective in disinfecting dentine specimens [Preston et al., 2007], but these and other methods, such as ethylene oxide and dry heat, may affect the properties of the dentine or its response to demineralisation [White et al., 1994; Preston et al., 2007]. Moreover, ethylene oxide may not be completely reliable for sterilising dentine [West et al., 2011].

Whichever disinfectant is selected, it is desirable to reduce the amount of soft tissue on the specimens, e.g. by brushing the outer surface of the tooth with water and by removing the pulp after separating the crown from the roots.

Most of the above-mentioned methods will not be effective against prions. A prion disease of particular concern is variant Creutzfeldt-Jacob disease (vCJD), which is transmissible from infected human or cattle tissues. The prevalence of VCJD in populations of both humans and cattle is low [Doerr et al., 2003; Walker et al., 2008]. Classification of bovine tissues according to risk of infection [Doerr et al.,2003] does not include teeth, but oral tissues from vCJD patients do not appear to contain prions [Walker et al., 2008]. The risk of prion transmission in an in situ experiment thus appears very small. Nevertheless, the occurrence of prions in the trigeminal ganglion of infected subjects and uncertainties regarding sensitivities of detection methods indicate that, as for dental treatment [Walker et al., 2008], it is wise to take precautions against prion infection from in situ specimens of dental tissue.

Several methods are available for inactivating prions [Canadian Food Inspection Agency, 2003; World Health Organization, 2003]. Of the low-temperature methods suitable for treating dental tissues, a 30-min immersion in sodium hypochlorite solution $(\mathrm{NaOCl})$ containing $\geq 16,500 \mathrm{ppm}$ available chlorine (approximately 5\% $\mathrm{NaOCl}$, as used in many domestic bleaches [World Health Organization, 2003]) should produce complete inactivation. Although $\mathrm{NaOCl}$ does not seem to affect enamel hardness, this agent could have a marked effect on dentine, since it can not only extract the organic matrix but also alter the mineral phase of the tissue [Sakae et al., 
1988]. An alternative to $\mathrm{NaOCl}$ might be immersion for $30 \mathrm{~min}$ in $1-2 \mathrm{~mol} / 1 \mathrm{NaOH}$ solution which, although considered to produce significant titre reduction rather than complete elimination [Canadian Food Inspection Agency, 2003], was found to remove prions from bovine bone graft material [Wenz et al., 2001]. Although $\mathrm{NaOH}$ may damage dentine less than $\mathrm{NaOCl}$, this needs to be confirmed experimentally.

To achieve the greatest effect against prions, teeth should be kept moist up to the time of treatment with the chosen agent.

\section{Erosive Challenge}

The challenge can be applied intra- or extra-orally. The ideal is intra-oral application, but ethical or other restraints may preclude this. Administration of the erosive challenge or agent can be supervised or unsupervised, but supervision is essential for greatest control and standardisation.

\section{Appliance Design}

Appliance design and location in the mouth are influenced by the need to prevent significant plaque build-up and, in erosion-only models, to protect specimens from abrasion [West et al., 2011]. Treatment of specimens with chlorhexidine ex vivo can be used to inhibit plaque accumulation [West et al., 1998]. Importantly, the oral appliance should be designed with subject safety in mind, especially to avoid the risk of choking, and the specimens should be secured against accidental loss. The positioning of the samples and the appliance should be consistent in all subjects. In the view of experts, there is no preferred design.

\section{Experimental Design}

Multiple cycle models were considered to be better than single-challenge models, provided clinically relevant parameters are employed. Both cross-over and parallel-group studies can be appropriate, although the cross-over design was recommended by the initial erosion group. To avoid potential carry-over, adequate washout periods, e.g. 1 week, should be included in cross-over designs. Sample sizes should be determined by appropriate power calculations.

In situ models are typically cycling models in which acid challenges are followed by, or alternate with, periods of exposure to intra-oral conditions during which remineralisation may occur. It is rare for only the acid challenge to take place intra-orally. Cycling models can investigate the net effect of alternating acid challenge and reminer- alisation on previously intact surfaces or can investigate the effect of intra-oral remineralisation on pre-softened surfaces.

As for in vitro cycling models, demineralisation and remineralisation conditions need to be defined, as does the sequence of acid challenging versus remineralisation/ treatment procedures.

Clinically relevant matters which require consideration include:

- Acid challenge: type, severity and duration;

- Time between challenges;

- Lead-in period. The duration of the lead-in period should be determined. The type of dentifrice or mouthwash to be used by subjects during lead-in (fluoride or non-fluoride) should be determined;

- Oral hygiene. The type of dentifrice and mouthwash used during the experiment by the subjects (non-fluoride or fluoride) should be defined as this will determine the background fluoride exposure;

- An accommodating or conditioning phase for the appliances or specimens should be considered. During this phase, pellicle can form, the specimen surface can adjust to intra-oral conditions and salivary flow rate can settle down after the initial stimulation induced by inserting the appliance. Although a period of $2 \mathrm{~h}$ seems necessary for pellicle to achieve its full thickness [Skjörland et al., 1995; Sonju Clasen et al., 1997] and in vitro studies suggest that an erosion-protective effect takes a similar time to develop [Wetton et al., 2006], it was thought unnecessary to specify a minimum conditioning period: an important requirement was to have the specimen in contact with the oral environment. Prolonged conditioning times can also potentially interfere with the implementation of an in situ study. For instance, in a study which commences at mid-morning, a 2-hour conditioning period would necessitate removal of the appliance for lunch immediately after conditioning. These considerations are particularly relevant for initial erosion models;

- Removal of appliances during eating, drinking and tooth brushing;

- Washout period between phases in cross-over models: 1 week is considered adequate.

\section{Human Subject Characteristics}

For in situ and in vivo studies, subject selection must be based on explicit exclusion and inclusion criteria which must be relevant to the protocol of the study. It is desirable to record and report relevant dental and medical information about the subjects: 
- Salivary flow rate (stimulated and unstimulated);

- Salivary buffer capacity;

- Fluoride history;

- Oral health status;

- Aspects of general health relevant to erosion;

- Use of medication which affects salivary flow, gastric reflux, eating disorders, unusual diet, etc.

\section{Validation}

For all models, the question needs to be asked whether they include all elements of the current understanding of the disease (content validity). On this basis, in vitro models are incomplete, but in situ and in vivo models do approach the natural process of softening. The concepts of construct validity and prediction validity may be useful approaches to assess validity [Nyvad et al., 2003; BergBeckhoff et al., 2008]. There is a smaller range of available theoretical predictions to use in testing erosion models than there is in caries research. For instance, severe erosion is relatively resistant to fluoride in its usual forms. Nevertheless, it is worth asking questions such as whether dose/response or exposure time/response effects can be demonstrated by a model.

\section{Erosion-Only Models}

\section{Initial Erosion Models (Enamel Softening)}

It was considered essential that in studies of this phase of erosion it should always be checked whether the effects of erosion have indeed been confined to softening, i.e. that no surface loss has occurred. Profilometry is one method for evaluating surface loss. In the method by Zero et al. [1982, 1998], a hardness tester with a Knoop diamond is used to make a series of 'indicator' indentations using small loads directly above the baseline indentations. Following the experiment, indentations are measured again. Decreased length or disappearance of an indicator indentation indicates the extent of enamel surface loss (if any) due to the erosive challenge. For loads of 2$20 \mathrm{~g}$, the depths of indicator indentations would be $0.21-$ $0.89 \mu \mathrm{m}$ [A. Lussi and D. Zero, unpubl. data], so extremely small losses of surface tissue can be detected. However, the accuracy of quantitative estimates of surface loss is unlikely to be high because of tissue loss within the indentation [Attin, 2006].

\section{In vitro Models}

It was considered that single-exposure models using evaluation by micro- or nanohardness are useful for screening products for erosive potential, although multiple-exposure models can offer some advantages, e.g. when products with an extremely wide range of erosive potential are tested [Barbour et al., 2011]. Single-exposure models can also be used for exploring systematically many of the factors which might be used to predict the erosive potential of soft drinks. Multiple-exposure models may involve either simply repeated acid exposures or cycles of short acid exposures alternating with exposure to saliva. The latter approach is considered to better reflect the erosive challenges faced by the dentition and is therefore encouraged. In both single- and multiple-exposure models, tooth specimens should ideally be pre-treated with saliva for pellicle formation.

\section{In situ Models}

To remain within the 'softened' state, specimens must be protected from both erosive wear and significant plaque build-up. These considerations impinge on appliance design. In some models, the severity and duration of the acid challenges or the number of challenges during a study cause surface loss simply through erosion [West et al., 1998]. In these studies, intra-oral administration of the challenge involves a risk to the dentition, and strict monitoring is required.

\section{In vivo Models}

Analyses of the $\mathrm{pH}$ and electrolyte concentrations of saliva offer useful information for in vivo studies by providing information on the severity and persistence of an erosive challenge. As the $\mathrm{pH}$ of saliva changes after collection through loss of $\mathrm{CO}_{2}$, samples should be collected under oil or by aspiration into a syringe [Jensdottir et al., 2005].

It is easy to soften enamel in vivo but in vivo erosion models await better assessment techniques. Areas where technical developments are needed include:

- Modifications of current models, e.g. development of split-mouth or split-tooth models [Young and Tenuta, 2011]. The principal aim here would be to improve the precision of measurements by improved pairing of treatment. However, in developing such models, it is important to be aware of the potential problem of carry-over;

- For in vivo acid challenges, it is desirable that acid challenges be localised to avoid exposing too large a part of the dentition to damage and also as part of developing split-tooth models. The use of gels to localise the acid challenge is likely to be inappropriate, as this tends to inhibit erosion almost completely [Shellis et 
al., 2005]. Some form of 'masking' to define test areas will therefore be required;

- More work on measuring the dynamics of tooth surface $\mathrm{pH}$ during an erosive challenge.

\section{Erosive-Wear Models}

\section{In vitro Models}

The physical component of the challenge will involve a rubbing action frequently, but not exclusively, involving tooth brushing. While tooth brushing may not always be clinically relevant to the site and location of the erosive wear, it is a convenient surrogate for intra-oral frictional forces. The force, the number of strokes, the type of brush, the length of time, the abrasive and any lubricant system should be controlled. The force used in published studies varies over a wide range. Based on a consideration of recent literature, Wiegand and Attin [2011] recommended a force of $2-3 \mathrm{~N}$, but since ISO 14569-1 82007 allows $0.5-2.5 \mathrm{~N}$, a force of $2 \mathrm{~N}$ might be a good recommendation. Wiegand and Attin [2011] provide clear guidance in choosing other brushing variables. The application of the agent must reflect the clinical situation.

\section{In situ Models}

The chosen mechanical component of wear should be clinically relevant, and the action could be either intra- or extra-oral. The oral appliance should be designed safely and the sample secured against accidental loss. The positioning of the samples and the appliance should be consistent in all subjects. In the view of experts, there is no preferred design.

\section{Clinical Trials}

The discussion focused on the feasibility of conducting clinical trials, given current knowledge of prevalence and incidence of erosion, and the currently available assessment methods. The ethical dimension was also touched on. For instance, the question of the most suitable treatment to offer participants was raised. An unavoidable complication involving any intervention study or one that investigates progression is the potential effect of dietary counselling. This is probably the best available treatment at present, so it was considered ethically appropriate to provide dietary counselling and unethical to withhold it. Once given, counselling could have an im- pact on a study, but this was considered an unavoidable complication in the ethical conduct of studies.

Epidemiological studies [Jaeggi and Lussi, 2006; Kreulen et al., 2010] suggest that the prevalence of erosion varies widely between different countries and different populations. A complicating factor in obtaining accurate estimates of prevalence is the variation between different indices or thresholds of severity.

It was pointed out that increase in erosion varies with age among adults. Adult studies, while as relevant as studies on children, are more challenging to perform unless 'captive' groups, such as armed forces or universities, are recruited. But these groups rarely reflect the general population and are therefore not ideal.

Children, particularly adolescents, provide a population that can conveniently be investigated within reasonable limits. From the point of view of assembling samples (schools) and of the type of wear, 12- to 16-year-olds seemed to be the most convenient population. The sample size for power calculations depends on which outcome variable progression or incidence - is to be used. In early adolescents, a sample size of 6,000 is required if progression is the outcome. On the other hand, it was suggested that, assuming a population erosion increment of $20 \%$ /year, an erosion study where the outcome variable is the incidence of new wear should be similar in size to a caries trial.

It was suggested that the clinical measure of erosion should be an index rather than a method such as profilometry, because at present the latter has not been fully validated [Schlueter et al., 2011]. However, it was considered essential that any index to be used in a clinical trial should, as far as possible, exclude attrition, e.g. by ignoring wear on incisal edges. Moreover, the index should differentiate between wedge-shaped defects and erosion. Such modifications should improve the ability to determine the effect of an anti-erosive treatment. It was emphasised that there was a strong need for good calibration of examiners. Models cast from impressions have been used successfully in some studies and could be useful in future clinical trials. However, diagnosis from models might be more difficult than from clinical examination of teeth, because of the absence of tooth colour and surface texture, so work to establish the accuracy of diagnosis from models is required. There was general agreement that erosion indices are as good as caries indices.

\section{Disclosure Statement}

The authors declare that they have no conflicts of interest in relation to this paper. 


\section{References}

Amaechi BT, Higham SJ, Edgar WM: Efficacy of sterilisation methods and their effect on enamel demineralisation. Caries Res 1998; 32:441-446.

Amaechi BT, Higham SJ, Edgar WM: The use of gamma irradiation for the sterilization of enamel for intra-oral cariogenicity tests. J Oral Rehabil 1999;26:809-813.

Attin T: Methods for assessment of dental erosion; in Lussi A (ed): Dental Erosion. From Diagnosis to Therapy. Monogr Oral Sci. Basel, Karger, 2006, vol 20, pp 152-172.

-Attin T, Meyer K, Hellwig E, Buchalla W, Lennon ÁM: Effect of mineral supplements to citric acid on enamel erosion. Arch Oral Biol 2003;48:753-759.

Barbour ME, Lussi A, Shellis RP: Screening and prediction of erosive potential. Caries Res 2011;45(suppl 1):24-32.

Barbour ME, Rees JS: The laboratory assessment of enamel erosion: a review. J Dent 2004;32: 591-602.

-Berg-Beckhoff G, Kutschmann M, Bardehle D: Methodological considerations concerning the development of oral dental erosion indexes: literature survey, validity and reliability. Clin Oral Invest 2008;12(suppl 1):51-58.

-Brauer DS, Saeki K, Hilton JF, Marshall GW, Marshall SJ: Effect of sterilization by gamma radiation on nano-mechanical properties of teeth. Dent Mater 2008;24:1137-1140.

Canadian Food Inspection Agency: Bovine spongiform encephalopathy agent - material safety data sheet for veterinary diagnostic laboratories, 2003 (http://www.msdsonline. com/CustomerSupport/Disease-MSDS/MSDSBovineSpongiformEncephalopathyAgent.pdf).

Carlén A, Börjesson AC, Nikdel K, Olsson J: Composition of pellicles formed in vivo on tooth surfaces in different parts of the dentition, and in vitro on hydroxyapatite. Caries Res 1998;32:447-455.

-Chandler NP: Preparation of dental enamel for use in intraoral cariogenicity experiments. J Dent 1990;18:54-58.

Doerr HW, Cinatl J, Rabenau HF: Prions and orthopedic surgery. Infection 2003;31:163-171.

- Fujikawa H, Matsuyama K, Uchiyama A, Nakashima S, Ujiie T: Influence of salivary macromolecules and fluoride on enamel lesion remineralization in vitro. Caries Res 2008;32:37-45.

-Hannig C, Berndt D, Hoth-Hannig W, Hannig $\mathrm{M}$ : The effect of acidic beverages on the ultrastructure of the acquired pellicle - an in situ study. Arch Oral Biol 2009;54:518-526.

-Hemingway CA, Shellis RP, Parker DM, Addy M, Barbour ME: Inhibition of hydroxyapatite dissolution by ovalbumin as a function of $\mathrm{pH}$, calcium concentration, protein concentration and acid type. Caries Res 2008;42: 348-353.
Holme B, Hove LH, Tveit AB: Using white light interferometry to measure etching of dental enamel. Measurement 2005;38:137-147.

Huysmans MC, Chew HP, Ellwood RP: Clinical studies of dental erosion and erosive wear. Caries Res 2011;45(suppl 1):60-68.

Jaeggi T, Lussi A: Prevalence, incidence and distribution of erosion; in Lussi A (ed): Dental Erosion. From Diagnosis to Therapy. Monogr Oral Sci. Karger, Basel, 2006, vol 20, pp 44-65.

Jensdottir T, Nauntofte B, Buchwald C, Bardow A: Effects of sucking acidic candy on wholemouth saliva composition. Caries Res 2005 39:468-474.

Kreulen CM, van 't Spijker A, Rodriguez JM, Bronkhorst EM, Creugers NH, Bartlett DW: Systematic review of the prevalence of tooth wear in children and adolescents. Caries Res 2010;44:151-159.

Koulourides T: Experimental changes of mineral density; in Harris RS (ed): Art and Science of Dental Caries Research. New York, Academic Press, 1968, pp 355-378.

Larsen MJ, Pearce EIF: Saturation of human saliva with respect to calcium salts. Arch Oral Biol 2003;48:317-322.

Lippert F, Parker DM, Jandt KD: Toothbrush abrasion of surface softened enamel studied with tapping mode AFM and AFM nanoindentation. Caries Res 2004;38:464-472.

Nyvad B, Machiulskiene V, Baelum V: Construct and predictive validity of clinical caries diagnostic criteria assessing lesion activity. J Dent Res 2003;82:117-122.

Preston KP, Higham SM, Smith PW: The efficacy of techniques for the disinfection of artificial sub-surface dentinal caries lesions and their effect on demineralization and remineralization in vitro. J Dent 2007;35:490-495.

Sakae T, Mishima H, Kozawa Y: Changes in bovine dentin mineral with sodium hypochlorite treatment. J Dent Res 1988;67:12291234.

Schlueter N, Hara A, Shellis RP, Ganss C: Methods for measurement and characterization of erosion in enamel and dentine. Caries Res 2011;45(suppl 1):13-23.

Schulz KF, Altman DG, Moher D, for the CONSORT Group: CONSORT 2010 Statement: updated guidelines for reporting parallel group randomised trials. Ann Intern Med 2010;152:726-732.

Shellis RP, Finke M, Eisenburger M, Parker DM, Addy M: Relationship between enamel erosion and flow rate. Eur J Oral Sci 2005;113: $232-238$.
Skjörland KK, Rykke M, Sonju T: Rate of pellicle formation in vivo. Acta Odontol 1995;53: 358-362.

Sonju Clasen AB, Hannig M, Skjörland KK, Sonju T: Analytical and ultrastructural studies of pellicle on primary teeth. Acta Odontol Scand 1997;55:339-343.

Viana PS, Machado AL, Giampaolo ET, Pavarina AC, Vergani CE: Disinfection of bovine enamel by microwave irradiation: effect on the surface microhardness and demineralization/remineralization processes. Caries Res 2010;44:349-357.

Walker JT, Dicjonson J, Sutton JM, Marsh PD, Raven NDH: Implications for CreutzfeldtJakob Disease (CJD) in dentistry: a review of current knowledge. J Dent Res 2008;87:511519.

Wenz B, Oesch B, Horst M: Analysis of the risk of transmitting bovine spongiform encephalopathy through bone grafts derived from bovine bone. Biomaterials 2001;22:15991606.

West NX, Davies M, Amaechi BT: In vitro and in situ erosion models for evaluating tooth substance loss. Caries Res 2011;45(suppl 1):4352.

West NX, Maxwell A, Hughes JA, Parker DM, Newcombe RG, Addy M: A method to measure clinical erosion: the effect of orange juice consumption on erosion of enamel. J Dent 1998;26:329-335.

Wetton S, Hughes JA, West NX, Addy M: Exposure time of enamel and dentine to saliva for protection against erosion: a study in vitro. Caries Res 2006;40:213-217.

Wetton S, Hughes JA, West NX, Newcombe RG, Addy M: The effect of saliva derived from different individuals on the erosion of enamel and dentine. A study in vitro. Caries Res 2007;41:423-426.

World Health Organization: Infection control guidelines for transmissible spongiform encephalopathies, 2003 (http://www.who.int/ csr/resources/publications/bse/whocdscsraph2003.pdf)

White JM, Goodis HE, Marshall SJ, Marshall GW: Sterilization of teeth by gamma radiation. J Dent Res 1994;73:1560-1567.

Wiegand A, Attin T: Design of erosion/abrasion studies - insights and rational concepts. Caries Res 2011;45(suppl 1):53-59.

-Young A, Tenuta LMA: Initial erosion models. Caries Res 2011;45(suppl 1):33-42.

Zero DT, Barillas I, Hayes AL, Fu J, Li H: Evaluation of an intraoral model for the study of dental erosion (abstract 132). Caries Res 1998;32:312-313

Zero DT, Curzon MEJ, Zander HA: Physical and chemical effects of toothpastes on dental enamel. J Dent Res 1982;61:451-455. 